\title{
Two-Step Validation in Character-based Ingredient Normalization
}

\author{
Jun Harashima and Yoshiaki Yamada \\ Cookpad Inc. \\ \{jun-harashima, yoshiaki-yamada\}@cookpad.com
}

\begin{abstract}
Although ingredients are important items of information in recipes, it is difficult to process them, especially for computers, because they are user-generated informal text. To normalize ingredients, we can use a character-based encoder-decoder model that takes the character sequence of an ingredient as an input and outputs its canonical form. However, the model still has two problems: The first is that the model often generates unnatural sequences as outputs. The second problem is that the generated sequences are sometimes unrelated to the original ingredient. Therefore, we propose a two-step validation to generate better normalizations. In the first validation step, we use a trie to limit the normalization candidates to existing sequences. In the second validation step, we rerank the normalization candidates based on their similarity to the original ingredient. We conducted experiments using a corpus that includes approximately 10 thousand pairs of ingredients and their canonical forms and showed that our proposed validation improved the performance of encoder-decoder models.
\end{abstract}

\section{CCS CONCEPTS}

- Computing methodologies $\rightarrow$ Natural language processing;

\section{KEYWORDS}

recipe, ingredient, normalization

\section{ACM Reference Format:}

Jun Harashima and Yoshiaki Yamada Cookpad Inc. \{jun-harashima, yoshiakiyamada\}@cookpad.com . 2018. Two-Step Validation in Character-based IngredientNormalization. In CEA/MADiMa'18: foint Workshop on Multimedia for Cooking and Eating Activities and Multimedia Assisted Dietary Management in conjunction with the 27th International foint Conference on Artificial Intelligence IfCAI, July 15, 2018, MÃd'ssvãd'gen, Stockholm, Sweden. ACM, New York, NY, USA, 5 pages. https://doi.org/10.1145/3230519.3230589

\section{INTRODUCTION}

Nowadays, many people upload their recipes to the Internet. For example, approximately 2.7 million recipes have been uploaded to Cookpad, one of the largest recipe services in the world. Thanks to such recipes, planning daily meals has become much easier.

However, it is difficult for computers to process these recipes because they are user-generated text. Consider the Japanese language as an example. The ingredient "corn" can be expressed as と

Permission to make digital or hard copies of part or all of this work for personal or classroom use is granted without fee provided that copies are not made or distributed for profit or commercial advantage and that copies bear this notice and the full citation on the first page. Copyrights for third-party components of this work must be honored. For all other uses, contact the owner/author(s).

CEA/MADiMa'18, July 15, 2018, Mässvägen, Stockholm, Sweden

(C) 2018 Copyright held by the owner/author(s).

ACM ISBN 978-1-4503-6537-6/18/07.

https://doi.org/10.1145/3230519.3230589
うもろこし,トーモロコシ, 玉蜀㯟, and so on, and it is up to the user which expression he or she uses in his or her recipes.

We can apply recent neural machine translation techniques [1, 8] to normalize these words. For example, character-based encoderdecoder models can be used to translate the original strings into their canonical forms [2, 7].

Nevertheless, we found that normalization methods based on encoder-decoder models have two drawbacks: The first is that they sometimes generate unnatural sequences (hereafter called the unnatural sequence problem). Even if the canonical form of トーモロ コシ is とうもろこし, the string can be normalized to とろろこし, which is an unnatural sequence.

The second drawback is that the methods often generate sequences that are dissimilar to their original string (hereafter called the dissimilar sequence problem). For example, トーモロコシ can be normalized to とろろこんぶ (tangle flakes). Of course, this is an incorrect normalization. Because the generated sequences are possible, this problem is more troublesome, especially in recipes.

In this paper, we propose a two-step validation to address these drawbacks. The first validation limits generated sequences using a trie, which is constructed from existing ingredients in recipes. This forces our decoder not to output unnatural sequences. In the second validation, we consider the similarities between the original string and its normalization candidates. We rerank the candidates by their similarity and select the one with the highest similarity. This ensures that the output is not dissimilar to the input.

\section{INGREDIENT NORMALIZATION}

Our task is to recognize a canonical form for each ingredient in a recipe. Figure 1 shows an ingredient list in a recipe from Cookpad, and we explain our task using this example.

We can see a variety of linguistic phenomena in this recipe. The red rectangle shows an example of a synonymous expression. Although this ingredient is represented by 豚ミンチ肉 (ground pork) in this recipe, it could be represented by 豚ひき肉 in other recipes. If the canonical form of this ingredient is defined as the latter one, our task is to normalize 豚ミンチ肉 to 豚ひき肉.

The green rectangle shows a phenomenon similar to synonymous expressions. This is an example of orthographic variants. This ingredient can be represented not only by 干ししいたけ (dried shiitake), but also by ほししいたけ. This phenomenon is a common problem in Japanese because the language has four different character types: hiragana, katakana, Chinese characters, and the Roman alphabet.

As seen in the orange rectangle, parentheses often appear at the end of ingredients. モロヘイヤ (葉だけ) means Jew's mallow (leaves only). In the figure, we can also see other examples (顆粒), (チューブ), and (炒め用), which are “(granules)“, “(tube)“, and “(for frying)", respectively. Because the information in parentheses is a 
CEA/MADiMa'18, July 15, 2018, Mässvägen, Stockholm, Sweden モロヘイヤたっぷり!ジュ

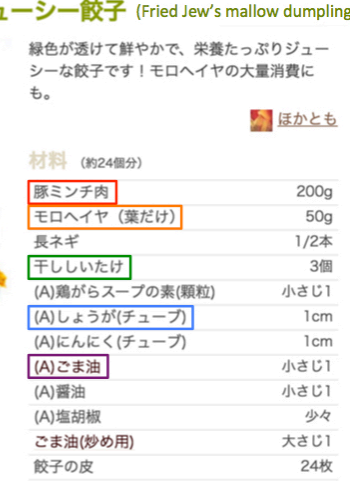

Figure 1: An example of a recipe from Cookpad.

supplementary explanation, we remove this text from the ingredients in this task.

Like terms in parentheses, symbols at the beginning of the ingredients are also removed in our task. For example, the text (A) ごま油 “(A) sesame oil” in the purple rectangle should be normalized to ごま油 (sesame oil). The symbols are mainly used to group ingredients and to refer them in recipes such as "mix (A) together." Other examples that often appear in recipes include $\bullet, *$, and $\star$.

As described above, many linguistic phenomena occur in this task. Furthermore, these phenomena occur simultaneously, as seen in the text in the blue rectangle. In this case, we address the problems of parentheses and symbols to normalize (A) しょうが (チュー ブ) “(A) ginger (tube)“ to しょうが (ginger).

\section{RELATED WORK}

Recently, text normalization has gradually begun to attract attention. Sasano et al. [6] introduced a variety of derivational patterns into Japanese morphological analysis to handle unknown words that are derived from words in a predefined lexicon. Using these patterns, they transformed informal expressions into standard ones and found the optimal analysis results for informal sentences.

Kaji and Kitsuregawa [3] not only used such hand-crafted patterns but also introduced a discriminative model to select the optimal results. Furthermore, they constructed a microblog corpus, in which informal words were annotated with their formal ones, to train the model and evaluate it using the corpus.

Although Saito et al.'s study [5] was similar to that of Kaji and Kitsuregawa, they automatically extracted derivational patterns from a training dataset that includes pairs of informal and formal expressions. Like Kaji and Kitsuregawa's study, the extracted patterns were used in their discriminative model.

Unlike the above studies, Ikeda et al. [2] purely focused on text normalization regardless of morphological analysis. They were the first to use an encoder-decoder model for Japanese text normalization and show its effectiveness through their experiments. We further explain their model in the next section.

For the English language, Sproat and Jaitly [7] also proposed a normalization method based on an encoder-decoder model for the text-to-speech task. In their model, while an input is a character sequence, an output is a word sequence. They also used finite-state transducers to restrict the possible outputs of their model.
Jun Harashima and Yoshiaki Yamada

Cookpad Inc.

\{iun-harashima, yoshiaki-yamada\}@cookpad.com

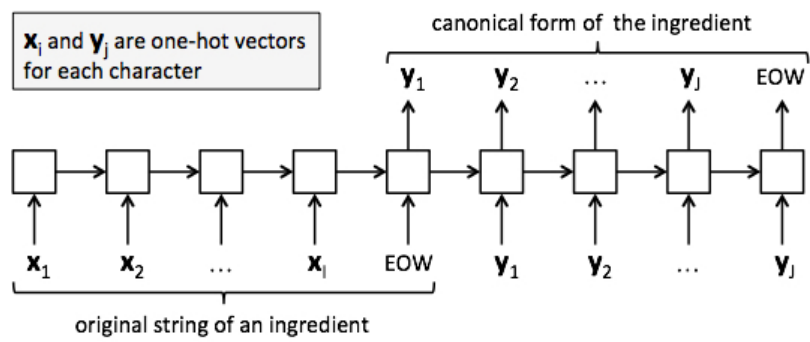

Figure 2: An overview of the character-based encoderdecoder method.

Unlike the studies of Sasano et al. [6], Kaji and Kitsuregawa [3], and Saito et al. [5], we do not take into account morphological analysis. Although Ikeda et al.'s study [2] is the basis of ours, we further introduce two validations into encoder-decoder models. The decoding process of Sproat and Jaitly's model [7] is different from ours because it is based on words.

\section{CHARACTER-BASED ENCODER-DECODER}

Like Ikeda et al.'s study [2], we use an encoder-decoder model for our task. An overview of this model is illustrated in Figure 2.

In our method, the encoder takes a string, which is an ingredient in this study, as an input and generates a vector for it. First, the string is split into characters and they are represented as one-hot vectors $\boldsymbol{x}_{i}(i=1,2, \ldots, I)$. Then, the vectors are fed to the encoder one by one and a single vector is finally created by the network.

The decoder generates a word, that is, a canonical form of the ingredient in this study, from the vector. The network outputs characters the represented by $\boldsymbol{y}_{j}(j=1,2, \ldots, J)$, which are also onehot vectors, until it outputs a special signal, EOW (End Of Word), as shown in Figure 2. We can obtain the final output by concatenating the characters.

In the decoding process, we can use a beam search to find better normalizations. In a beam search, the decoder has $N$ candidate sequences at each time step, outputs $M$ characters for each candidate, and selects the $N$ candidates with the highest probabilities from the $N \times M$ choices for the next time step. Using a beam search, we can find the globally optimal normalization.

\section{PROPOSED VALIDATION}

Even if a beam search is used in the decoder, there are still two problems: the unnatural sequence problem and dissimilar sequence problem. To address these problems, we propose two validations in the decoding process: trie-based limitation and similarity-based reranking.

\subsection{Trie-based Limitation}

As described in Section 1, encoder-decoder models sometimes generate unnatural sequences such as unnatural sentences in machine translation or unnatural words in our task.

Consider the top part of Figure 3. In this example, the original string is こめ油 (rice bran oil) and its canonical form is 米油. Here, $\boldsymbol{x}$ and $\boldsymbol{y}$ represent the original string and its normalized candidate, respectively. Probablity $\operatorname{prob}(\boldsymbol{y} \mid \boldsymbol{x})$ is calculated in the decoding process. The encoder-decoder model described in the previous 


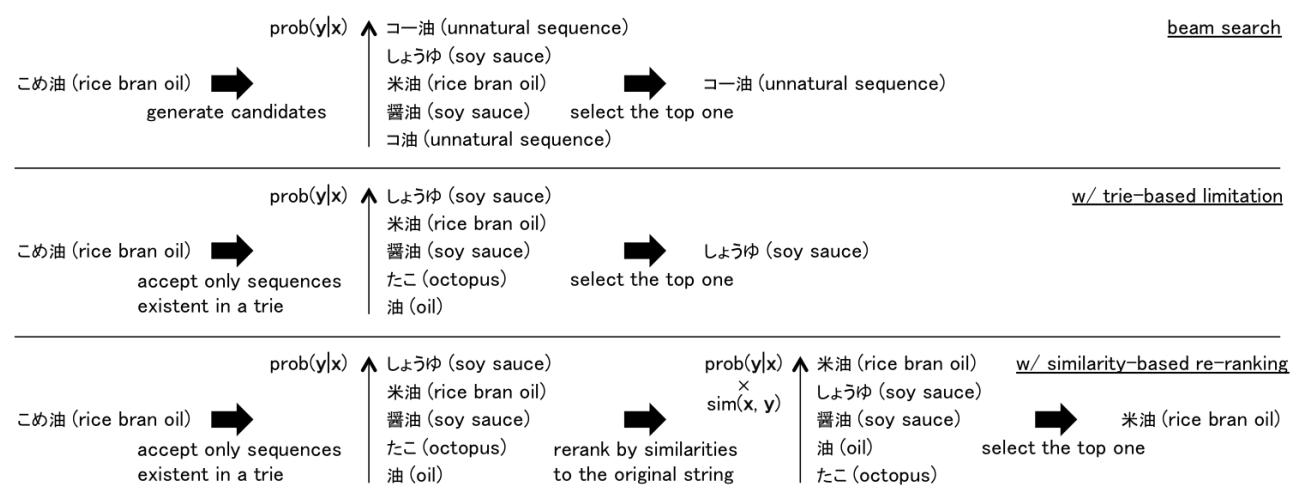

Figure 3: Overviews of a beam search, trie-based limitation, and similarity-based reranking.

section wrongly outputs コー油, which is an unnatural sequence, even if the decoder uses a beam search.

To tackle the unnatural sequence problem, we introduce a triebased limitation into the decoding process. As is well known, a trie is a data structure that can be used to store words. First, we construct a trie in advance from ingredients in existing recipes. The trie allows us to know whether a character sequence exist in the ingredients.

We then limit characters in each decoding step so that the sequences with the characters exist in the trie. Consider the top and middle parts of Figure 3. Here, コー油 and コ油 are rejected in our proposed validation because they do not exist in the trie.

More precisely, characters that generate impossible sequences are rejected as $\boldsymbol{y}_{j}$. Suppose that $\boldsymbol{y}_{1}$ and $\boldsymbol{y}_{2}$ are $\sqsupset$ and - , respectively. Our validation does not allow the encoder-decoder model to set $\boldsymbol{y}_{3}$ to 油 because $コ$ 一油 is an unnatural sequence, as described above, so it looks for another character for $\boldsymbol{y}_{3}$.

\subsection{Similarity-based Reranking}

Although our trie-based validation accepts only natural sequences, we still have the dissimilar sequence problem. That is, candidate sequences that exist in our trie can pass the first validation even if they are dissimilar to the original string.

Consider again the middle part of Figure 3. Our trie-based validation still accepts candidate sequences such as しょうゆ (soy sauce), 酱油 (soy sauce), and たこ (octopus), which are dissimilar to the original string こめ油 (rice bran oil).

We thus take into account similarities between the original string and its normalization candidates. As in the example, しょうゆ, 酱 油, and ここ are reranked lower and 米油 and 油 (oil) are reranked higher if their similarities to こめ油 are calculated. For example, we can use a word2vec model [4] to calculate such similarities.

More precisely, we rerank the candidate sequences using the following function:

$$
\operatorname{score}(\boldsymbol{x}, \boldsymbol{y})=\operatorname{prob}(\boldsymbol{y} \mid \boldsymbol{x}) * \operatorname{sim}(\boldsymbol{x}, \boldsymbol{y}),
$$

where $\operatorname{sim}(\boldsymbol{x}, \boldsymbol{y})$ represents the similarity between $\boldsymbol{x}$ and $\boldsymbol{y}$.

Using not only the trie-based limitation but also similarity-based reranking, we can generate a natural sequence that is similar to the original string.

\section{EXPERIMENTS}

\subsection{Data}

We collected 10,599 distinct ingredients from recipes in Cookpad and normalized them manually. We divided the pairs of the ingredients and their normalized versions into training, validation, and test sets. The number of pairs in each set were $8,479,1,060$, and 1,060 , respectively. The training set was used to train our machine learning-based methods, the validation set was used to tune them, and the test set was used to evaluate all methods described in the next section.

\subsection{Methods}

In our experiments, we compared the outputs of the following methods.

AsItIs This method performed no normalization. That is, it outputted each input as it was.

RegExp A simple regular expression-based method, which removes symbols and parentheses from the beginning and the end of each input, respectively.

EncDec The character-based encoder-decoder model described in Section 4.

Beam The EncDec method with a beam search, also described in Section 4.

Trie A beam search with our trie-based limitation described in Section 5.1.

Sim A trie-based model with our similarity-based reranking described in Section 5.2.

For EncDec, Beam, Trie, and Sim, we used stacked long shortterm memories both in the encoder and decoder. We set the number of layers to 3, the number of units to 1,024 , and parameters $N$ and $M$ described in Section 4 to 10 . These values were set according to the results of our validation set. We did not use an attention mechanism because ingredients were short and the method was not effective in our preliminary experiments.

We did not limit the size of vocabularies for source and target sides. That is, we used all characters in the ingredients and their normalized versions in our training data. The size of these vocabularies were 1,071 and 579, respectively. 
For Trie, we used the ingredients in all recipes that have been stored to Cookpad by August 2017 to construct our trie. Note that we ignored ingredients that appeared only one time in the recipes.

We also used the ingredients to train a word2vec model to enable Sim to calculate the similarities between the original strings and their normalization candidates. In the training, an ingredient was treated as a word and a sequence of ingredients (see Figure 1) was treated as a document. The settings of the model were their default values. That is, the training algorithm was a continuous bag-of-words, the number of units was set to 100 , and the size of the window was set to 5 .

\subsection{Results and Discussion}

Table 1 gives the results. Note that each accuracy represents a success rate that was calculated based on the number of exact matches between the system outputs and human normalizations. Additionally, $\dagger$ and $\ddagger$ indicate statistically significant improvements over EncDec and Beam with $p<0.01$ (sign test), respectively.

Because the accuracy of AsItIs was $20.8 \%$, we can say that approximately $20 \%$ of the ingredients in our test set did not need to be normalized. That is, their canonical forms are the same as the original strings. For example, the canonical form of キャベツ (cabbage) was キャベツ. Conversely, approximately $80 \%$ of ingredients in our test set needed to be normalized.

The naive regular expressions were not very effective because the accuracy of RegExp was about 30\% at most. Although RegExp removed unnecessary symbols and parentheses from the original strings, the method could not solve more complex problems such as synonymous expressions and orthographic variants. There was a limit to what the regular expressions could do.

EncDec could generate arbitrary string and thus, it performed

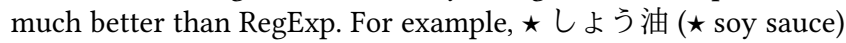
was successfully normalized to しょうゆ (soy sauce). Although this example contained a symbol issue and an orthographic variants issue, the method could solve both problems. We conclude that the encoder-decoder model was an effective solution for our task.

As we expected, the beam search improved the outputs of EncDec. For example, EncDec wrongly normalized トーモロコシ (corn) to とろろこし, which is not a natural sequence. In contrast, Beam could find the correct result とうもろこし (corn) by exploring normalization candidates with high probabilities at each time step.

We can see that Trie achieved higher performance than Beam. As described in Section 5.1, Trie selects only possible characters for each candidate sequence in the beam search. Suppose that $お$ 好みで柚子胡椒 (yuzukosho to taste) was the original string. Although the (incorrect) output of Beam was ゆでこしょう, which is an unnatural sequence, that of Trie was correct: ゆずこしょう (yuzukosho). In this case, since there were no ingredients beginning with ゆでこし in our recipes, our validation rejected impossible sequences at the beginning of the string and found the optimal output among other possible sequences.

Additionally, Sim achieved higher performance than Trie. For example, Trie wrongly normalized 餅粉 (glutinous rice flour) to でん (a type of Japanese stew), which was a natural but dissimilar sequence to the original string. In fact, おでん had a low similarity of 0.41 to 餅粉. In contrast, もち粉 (glutinous rice flour) had a
Table 1: Experimental results.

\begin{tabular}{ll}
\hline Method & Accuracy \\
\hline AsItIs & 20.8 \\
RegExp & 28.6 \\
EncDec & 71.2 \\
Beam & $72.3 \dagger$ \\
\hline Trie & $72.8 \dagger \dagger$ \\
Sim & $73.3 \dagger \dagger$ \\
\hline
\end{tabular}

high similarity 0.94 to the original string. Thus, Sim reranked the candidate as the top one.

Although our two validations were effective, there were still normalization errors. One of the most typical errors was due to a lack of training examples. For example, 桂皮 (cinnamon) was wrongly normalized to 陳皮 (chenpi) although it should have been normalized to シナモン (cinnamon). Because we had no training examples for シナモン in our training set, we had no choice but to add these examples to address this error.

In contrast, there were also errors relating to our two validations such as ハクサイ (Chinese cabbage), which was normalized to 空芯菜 (water spinach) although the string should be normalized into 白菜. This was because the similarity of 空芯菜 with 八 クサイ was higher than that of 白菜 in our recipes. Although the two validations improved performance, there is room for exploring better methods for calculating similarities.

\section{CONCLUSION}

In this paper, we introduced a novel task called ingredient normalization and addressed it based on a character-based encoderdecoder model. Furthermore, we proposed a two-step validation in the decoding process: The trie-based limitation forced the model to generate only natural normalization candidates. The similaritybased reranking caused the model to select a candidate with a high similarity to the original string. Our experimental results show that the two validations improved a simple encoder-decoder model with a beam search. In future work, we plan to explore better methods of calculating the similarities between an original string and its normalization candidates.

\section{REFERENCES}

[1] Kyunghyun Cho, Bart van Merriënboer, Caglar Gulcehre, Dzmitry Bahdanau, Fethi Bougares, Holger Schwenk, and Yoshua Bengio. 2014. Learning Phrase Representations using RNN Encoder-Decoder for Statistical Machine Translation. In Proceedings of EMNLP 2014. 1724-1734.

[2] Taishi Ikeda, Hiroyuki Shindo, and Yuji Matsumoto. 2017. Japanese Text Normalization with Encoder-Decoder Model. In Proceedings of WNUT 2017. 118-126.

[3] Nobuhiro Kaji and Masaru Kitsuregawa. 2014. Accurate Word Segmentation and POS Tagging for Japanese Microblogs: Corpus Annotation and Joint Modeling with Lexical Normalization. In Proceedings of EMNLP 2014. 99-109.

[4] Tomas Mikolov, Ilya Sutskever, Kai Chen, Greg Corrado, and Jeffrey Dean. 2013. Distributed representations of words and phrases and their compositionality. In Proceedings of NIPS 2013. 3111-3119.

[5] Itsumi Saito, Kugatsu Sadamitsu, Hisako Asano, and Yoshihiro Matsuo. 2014 Morphological Analysis for Japanese Noisy Text based on Character-level and Word-level Normalization. In Proceedings of COLING 2014. 1773-1782.

[6] Ryohei Sasano, Sadao Kurohashi, and Manabu Okumura. 2013. A Simple Approach to Unknown Word Processing in Japanese Morphological Analysis. In Proceedings of IJCNLP 2013. 162-170.

[7] Richard Sproat and Navdeep Jaitly. 2016. RNN Approaches to Text Normalization: A Challenge. ArXiv e-prints (2016). arXiv: 1611.00068

[8] Ilya Sutskever, Oriol Vinyals, and Quoc V. Le. 2014. Sequence to Sequence Learning with Neural Networks. In Proceedings of NIPS 2014. 3104-3112. 